\title{
Degeneración nodular de Salzmann en paciente mujer de 20 años de edad: un reporte de caso
}

\section{Salzmann's nodular degeneration in a 20-year old female patient: case report}

Correspondencia

Andres Herrera-Hurtado anderh021@gmail.com

\section{Recibido: 30/05/2019}

Arbitrado por pares

Aprobado: 21/08/2019

Citar como: Garcia-Rodriguez T, Herrera-Hurtado A, Burga-Kuroda H. Degeneración nodular de Salzmann en paciente mujer de 20 años de edad: un reporte de caso. Acta Med Peru. 2019;36(3):231-4

\author{
Tatiana Garcia-Rodriguez', Andres Herrera-Hurtado ${ }^{1}$, Harumi Burga-Kuroda²,a \\ 1 Facultad de Medicina, Universidad Nacional de Trujillo. Trujillo, Perú. \\ 2 Universidad Católica Nuestra Señora de la Asunción. La Asunción, Argentina. \\ a Especialista en Oftalmología
}

\section{RESUMEN}

La degeneración nodular de Salzmann es un trastorno corneal no inflamatorio, infrecuente, de mayor prevalencia en mujeres mayores de 50 años. Se caracteriza por la presencia de nódulos blanquecinos en la córnea y está asociada a diversas patologías. Reportamos el caso de una paciente de 20 años con historia de astigmatismo, miopía y síndrome de ovario poliquístico. Acudió a consulta por presentar sensación de cuerpo extraño en el ojo izquierdo y negó el haber padecido infección o trauma ocular recientes. Al examen oftalmológico se evidenció disfunción de las glándulas de Meibomio y nódulos opacos en la periferia de ambas córneas. Los hallazgos clínicos y la tomografía de coherencia óptica confirmaron el diagnóstico de degeneración nodular de Salzmann. El tratamiento con ciclosporina tópica demostró ser efectivo en la disminución de la inflamación causada por la disfunción de las glándulas de Meibomio, lo que evitó recurrencias nodulares a corto plazo.

Palabras clave: Enfermedades de la córnea; Glándulas de Meibomio; Síndrome de ovario poliquístico; Tomografía de coherencia óptica (fuente: DeCS-BIREME).

\section{ABSTRACT}

Salzmann's nodular degeneration is an uncommon non-inflammatory corneal disorder, which is more prevalent in women more than 50 years old. It is characterized by the presence of whitish nodules in the cornea and it is associated to certain conditions. We report the case of a 20-year old female patient with past history of astigmatism, myopia, and polycystic ovary syndrome. She presented reporting a foreign body sensation in her left eye and she denied having had recent eye infection or trauma. The ophthalmological examination revealed Meibomian gland dysfunction and opaque nodules in the periphery of both corneas. Clinical findings and an optical coherence tomography (OCT) corroborated the diagnosis of Salzmann's nodular degeneration. Therapy using topical cyclosporin proved to be effective in reducing the inflammation caused by Meibomian gland dysfunction, which prevented nodular recurrence in the short term.

Keywords: Corneal diseases; Meibomian glands; Polycystic ovary syndrome; Tomography, optical coherence (source: MeSH NLM). 


\section{INTRODUCCIÓN}

La degeneración nodular de Salzmann (DNS) es un trastorno corneal de carácter no inflamatorio, infrecuente y de progresión lenta. Clínicamente, se presenta en forma de nódulos de color blanquecino opaco o gris azulado, en diferentes tamaños (1-3 $\mathrm{mm}$ ) y que pueden estar aislados o agrupados en cualquier lugar de la córnea ${ }^{[1]}$. Los nódulos corresponden a una acumulación de tejido conectivo denso con desorganización de las laminillas de colágeno; se ubican debajo del epitelio corneal, lo que provoca su elevación y adelgazamiento de este último, además de la potencial desintegración de la membrana de Bowman. Suelen ser avasculares; sin embargo, en algunos casos, pueden presentar vascularización subepitelial ${ }^{[2,3]}$.

El origen de la enfermedad es idiopático. No obstante, su aparición ha sido asociada con la presencia de lesiones traumáticas debido al uso de lentes de contacto o el padecimiento de infecciones latentes. Otros procesos inflamatorios como queratitis intersticial, queratoconjuntivitis vernal, queratoconjuntivitis flictenular, uveítis crónica, distrofias corneales, tracoma y disfunción de las glándulas de Meibomio (DGM) también podrían desencadenar la DNS ${ }^{[4]}$. Además, se han involucrado factores genéticos -mediante patrones de herencia autosómica dominante- $y$ ambientales - a través de la continua exposición a la luz solar o irritantes externos- ${ }^{[1]}$.

Aunque la DNS suele ser asintomática, los pacientes pueden experimentar-según la ubicación de los nódulos- disminución de la agudeza visual, sensación de cuerpo extraño, ardor, picazón o enrojecimiento. Además, puede presentarse de forma unilateral o bilateral. Suele ser más frecuente en personas mayores de 50 años, especialmente en mujeres ${ }^{[5,6]}$.

Salzmann publicó el primer caso de DNS en el año $1925^{[7]}$; desde entonces poco se ha investigado al respecto y los casos reportados son escasos. Nuestro objetivo es presentar el diagnóstico y manejo de una paciente de 20 años con DNS bilateral periférica.

\section{REPORTE DE CASO}

Se presenta el caso de una paciente mujer de 20 años de edad con astigmatismo, usuaria de lentes oculares correctores para la miopía desde los 7 años; con diagnóstico de síndrome de ovario poliquístico desde los 14 años.

La paciente acudió al consultorio de Oftalmología por sensación de cuerpo extraño en el ojo izquierdo de dos días de evolución, sin disminución de la agudeza visual. Negó antecedente de lesión o infección ocular reciente. Al examen oftalmológico se encontró una agudeza visual de 20/50 en ambos ojos; asimismo, se evidenció resequedad de la superficie ocular, conductos de las glándulas de Meibomio obstruidos y disminución de su secreción al aplicar presión digital en los tarsos. El resto de anexos, medios y fondo de ojo no presentaron alteración. A la biomicroscopía y posterior tinción con fluoresceína se observaron lesiones nodulares blanquecinas de aspecto hialino ubicadas en la mitad temporal inferior de la córnea derecha y la mitad nasal inferior de la córnea izquierda (Figura 1).
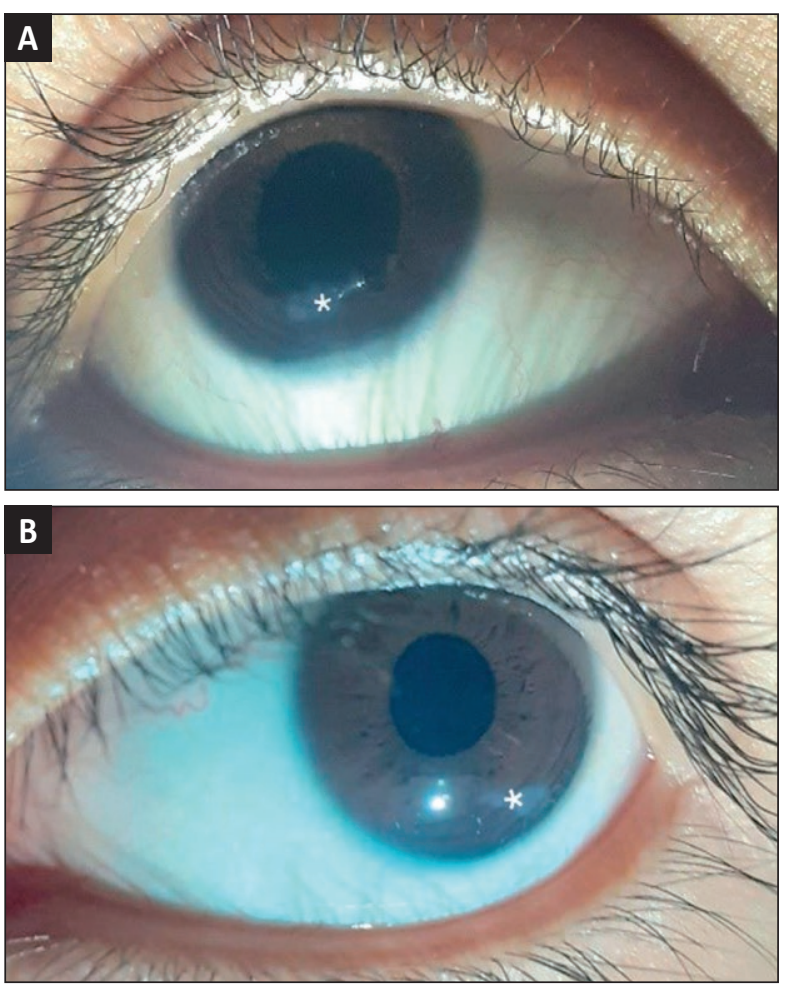

Figura 1. Fotografía del segmento anterior del ojo. Los asteriscos blancos marcan las lesiones blanquecinas de aspecto hialino que se encuentran localizadas en las regiones temporal inferior de la córnea derecha (A) y nasal inferior de la córnea izquierda (B).

Se inició tratamiento con una gota de fluorometolona 0,1\% cada 8 horas, durante tres semanas. Además, se aplicó un lubricante libre de preservantes y compresas de agua tibia para complementar el manejo. Al finalizar el periodo, se realizó una tomografía de coherencia óptica (TCO) del segmento anterior que evidenció lesiones hiperdensas entre el epitelio corneal y la membrana de Bowman en ambos ojos (Figura 2).
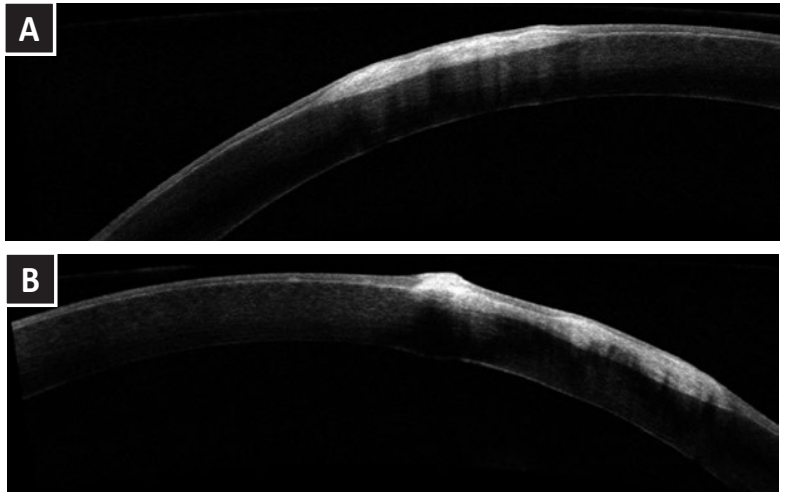

Figura 2. Tomografía de coherencia óptica del segmento anterior. Se muestra material hiperdenso entre el epitelio corneal elevado y la membrana de Bowman, que presenta adelgazamiento y discontinuidad. (A) ojo derecho y (B) ojo izquierdo. 
Los hallazgos clínicos y la TCO confirmaron el diagnóstico de DNS, por lo que se dio la indicación de una gota de ciclosporina 0,1\% cada 12 horas. Dos semanas después, la paciente manifestó ardor, por lo que se cambió la concentración de ciclosporina a 0,05\%. Tras doce semanas de tratamiento, al examen oftalmológico se observó disminución del tamaño de las lesiones, las cuales eran planas y no generaban molestias. Se retiró la ciclosporina y se continuó con el uso de lubricante para tratar la resequedad. Al control en tres meses, la paciente no refirió molestias ni disminución de la agudeza visual.

\section{DISCUSIÓN}

La DNS es un trastorno corneal infrecuente, de mayor prevalencia en mujeres mayores de 50 años, asociado a un daño corneal previo ${ }^{[8]}$; características que no se corresponden con nuestra paciente, quien tenía 20 años de edad y no tenía antecedentes de lesión traumática o infección ocular reciente.

Diversas patologías han sido relacionadas con la DNS. Hamada et al. encontraron que la mayoría de casos de DNS se presentaron en pacientes con DGM ${ }^{[5]}$. Nuestra paciente presentó obstrucción de los conductos de las glándulas, lo que es sugerente de la disfunción. Adicionalmente, se ha propuesto que en pacientes con síndrome de ovario poliquístico, como nuestra paciente, los altos niveles de andrógenos promoverían la obstrucción de los conductos de las glándulas, produciendo disminución de la secreción de lípidos de la película lagrimal, hiperosmolaridad y ruptura prematura de las lágrimas ${ }^{[9,10]}$.

La DGM produce desecación ocular que causa descamación y proliferación del epitelio corneal. Esta condición promueve el crecimiento excesivo de fibroblastos con el consiguiente aumento de fibras de colágeno y sobreexpresión de metaloproteinasas de matriz, características encontradas a nivel del epitelio de los nódulos de pacientes con DNS ${ }^{[1]}$.

El diagnóstico de la DNS engloba hallazgos clínicos, histológicos e imagenológicos. En este último caso se utiliza la TCO o la microscopía confocal ${ }^{[12]}$. La TCO ha demostrado tener una eficacia similar a la biopsia para el diagnóstico de la DNS sin necesidad de recurrir a procedimientos invasivos ${ }^{[13]}$. Nuestra paciente presentó los nódulos blanquecinos en la córnea característicos de la DNS que, al observarlos con la TCO, se ubicaban entre el epitelio corneal y la membrana de Bowman, hallazgo que corroboró el diagnóstico.

La localización y ubicación de los nódulos son determinantes para la elección del tratamiento. En los casos donde los nódulos interrumpen el eje visual, la queratectomía fototerapéutica con láser es considerada la mejor opción terapéutica; si la lesión produce infiltración de las membranas profundas, se puede realizar una queratoplastia ${ }^{[12,14]}$. Empero, cualquiera sea el método elegido, la frecuencia de recidivas es alta ${ }^{[5]}$. En nuestra paciente, por la ubicación periférica de los nódulos, se optó por un tratamiento conservador con ciclosporina tópica que también aportó al manejo de la DGM. Esta ha demostrado tener resultados positivos en la disminución de la inflamación ocular y la producción lagrimal [15]. De igual manera, aunque los nódulos disminuyeron de tamaño fue necesario mantener control de la paciente para evitar recurrencias.

La DNS sigue siendo una patología poco reportada. Se informó el caso de una paciente joven con síndrome de ovario poliquístico que presentó DNS asociado a DGM. El diagnóstico fue corroborado clínicamente y con la TCO. El tratamiento con ciclosporina tópica demostró ser efectivo en la disminución de recurrencias nodulares a corto plazo y atenuar los efectos de la disfunción glandular.

Contribuciones de autoría: todos los autores contribuyeron de manera activa, de acuerdo a los criterios de autoría en una publicación científica.

Fuente de financiamiento: la presente investigación no recibió financiamiento de agencias del sector público, sector comercial o entidades sin ánimo de lucro.

Conflicto de intereses: ninguno que declarar.

\section{REFERENCIAS BIBLIOGRÁFICAS}

1. Maharana PK, Sharma N, Das S, Agarwal T, Sen S, Prakash G, et al. Salzmann Nodular Degeneration. Ocul Surf. 2016;14(1):20-30.

2. Das S, Link B, Seitz B. Salzmann nodular degeneration of the cornea: A review and case series. Cornea. 2005;24(7):772-7.

3. Farjo AA, Halperin GI, Syed N, Sutphin JE, Wagoner MD. Salzmann nodular corneal degeneration clinical characteristics and surgical outcomes. Cornea. 2006;25(1):11-5.

4. Roszkowska AM, Wylegala E. Corneal Degeneration. In: Babizhayev $M$, Wan-Cheng Li D, editors. Studies on the Cornea and Lens (Oxidative Stress in Applied Basic Research and Clinical Practice). New York: Springer Science; 2015. p. 23-39.

5. Hamada S, Darrad K, McDonnell PJ. Salzmann nodular corneal degeneration (SNCD): Clinical findings, risk factors, prognosis and the role of previous contact lens wear. Cont Lens Anterior Eye. 2011;34(4):173-8.

6. Graue-Hernández EO, Mannis MJ, Eliasieh K, Greasby TA, Beckett LA, Bradley JC, et al. Salzmann nodular degeneration. Cornea. 2010;29(3):283-9.

7. Salzmann, M. I. Über eine Abart der knötchenförmigen Hornhautdystrophie. Ophthalmologica. 1925;57(1-6):92-9.

8. Turgut B, Çatak O, Demir T. Meibomian gland dysfunction: an overlooked eyelid disease. Adv Ophthalmology Vis Syst. 2018;8(3):168-72.

9. Baser G, Yildiz N, Calan M. Evaluation of Meibomian gland dysfunction in polycystic ovary syndrome and obesity. Curr Eye Res. 2017;42(5):661-5.

10. Bonini S, Mantelli F, Moretti C, Lambiase A, Bonini S, Micera A. Itchydry eye disease with polycystic ovary syndrome. Am J Ophthalmol. 2007;143(5):763-71. 
11. Eberwein P, Hiss S, Auw-Haedrich C, Sundmacher R, Hauer K, Boehringer D, et al. Epithelial marker expression in Salzmann nodular degeneration shows characteristics of limbal transient amplifying cells and alludes to an involvement of the epithelium in its pathogenesis. Acta Ophthalmol. 2010;88(5):e184-9.

12. Bernal DL, Almaguer IMG, Santos II, Yero A. Degeneración nodular de Salzmann. Rev Cuba Oftalmol. 2016;29(3):574-80.

13. Hurmeric V, Yoo SH, Karp CL, Galor A, Vajzovic L, Wang J, et al. In vivo morphologic characteristics of Salzmann nodular degeneration with ultra-high-resolution optical coherence tomography. Am J Ophthalmol. 2011;151(2):248-56

14. Paranjpe V, Galor A, Monsalve P, Dubovy SR, Karp CL. Salzmann nodular degeneration: prevalence, impact and management strategies. Clin Ophthalmol. 2019;13:1305-14.

15. Karpecki P, Shechtman D. A Look at Salzmann's [Internet]. Newtown Square, PA: Review of Optometry; 2011 [citado el 27 de agosto del 2019]. Disponible en: https://www.reviewofoptometry.com/ article/a-look-at-salzmanns 